\title{
Kinematic Modeling of Separation Compression for Paired Approaches to Closely-Spaced Parallel Runways
}

\author{
Michael M. Madden * \\ NASA Langley Research Center, Hampton, VA, 23681
}

\begin{abstract}
In a simultaneous paired approach to closely-spaced parallel runways, a pair of aircraft flies in close proximity on parallel approach paths. The longitudinal separation between the aircraft must be maintained within a range that avoids wake encounters and, if one of the aircraft blunders, avoids collision. To increase operational availability, the approach procedure must accommodate a mixture of aircraft sizes and, consequently, approach speeds. In these procedures, the slower aircraft is placed in the lead position. The faster aircraft maintains separation from the slow aircraft in a dependent operation until final approach and flies independently afterward. Due to the higher approach speed of the fast aircraft, longitudinal separation will decrease during final approach. Therefore, the fast aircraft must position itself before the final approach so that it will remain within the safe range of separation as separation decreases. Given the approach geometry and speed schedule for each aircraft, one can use kinematics to estimate the separation loss between a pair of aircraft. A kinematic model can complement fasttime Monte-Carlo simulations of the approach by enabling a tailored reduction in the variation of starting position for the fast aircraft. One could also implement the kinematic model in ground-based or on-board decision support tools to compute the optimal initial separation for a given pair of aircraft. To better match the autocoupled flight of real aircraft, the paper derives a kinematic model where the speed schedule is flown using equivalent airspeed. The predicted time of flight using the equivalent airspeed kinematic model compares well against a high-fidelity aircraft simulation performing the same approach. This model also demonstrates a modest increase in the predicted loss of separation when contrasted against a kinematic model that assumes the scheduled speed is true airspeed.
\end{abstract}

\section{Nomenclature}

$\gamma \quad=$ glideslope angle (radians)

$\rho=\quad=$ atmospheric density at altitude (slug per cubic foot)

$\rho_{0} \quad=$ atmospheric density at sea-level (slug per cubic foot)

ADS-B = Automatic Dependent Surveillance-Broadcast

a $\mathrm{EAS}_{\mathrm{A}}=$ constant acceleration of equivalent airspeed, negative on approach $(\mathrm{knot} / \mathrm{s})$

AGL $\quad=$ above ground level

$\mathrm{a}_{\text {slow }}=$ acceleration of the slow aircraft, negative on approach (knot/s)

CSPO $=$ Closely Spaced Parallel Operations

$\mathrm{d}_{\text {compress }}=$ compression of longitudinal separation as the slow aircraft travels from FAF to PTS

$\mathrm{D}_{\mathrm{CS}} \quad=$ collision safe distance (feet)

$\mathrm{D}_{\mathrm{EAS}} \quad=$ equivalent airspeed distance (feet)

$\mathrm{D}_{\text {decel }}=$ true or $\mathrm{D}_{\text {EAS }}$ distance traveled by the aircraft during deceleration (feet)

$\mathrm{D}_{\text {I-fast }}=\mathrm{D}_{\text {EAS }}$ of the fast aircraft from PTF when the slow aircraft is at the FAF and deceleration is independent (feet)

$\mathrm{D}_{\mathrm{D} \text {-fast }}=\mathrm{D}_{\text {EAS }}$ of the fast aircraft from PTF when the slow aircraft is at the FAF and deceleration is

\footnotetext{
* Chief Scientist, Simulation Development and Analysis Branch, Mail Stop 125B, Senior Member AIAA.
} 


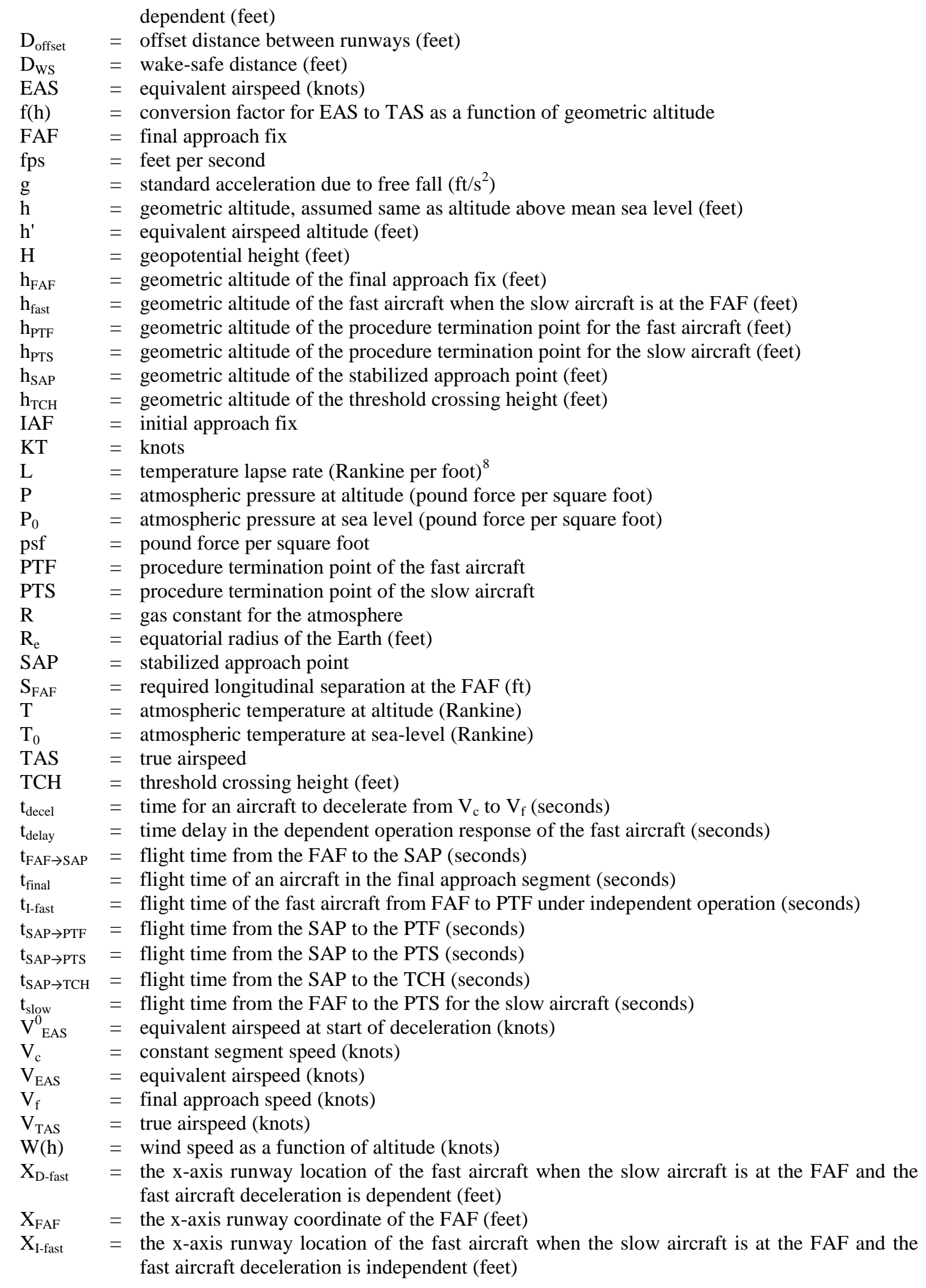




\section{Introduction}

$\mathrm{T}$ HE Closely Spaced Parallel Operations (CSPO) Working Group of the Federal Aviation Administration is investigating operational concepts to allow simultaneous paired approaches under instrument conditions at runways separated by as little as $700 \mathrm{ft}^{1,2}$ In a simultaneous paired approach, a pair of aircraft flies in close proximity to each other as they approach a pair of parallel runways. The longitudinal separation between the aircraft must be maintained within a range to avoid wake encounters and, if one of the aircraft blunders ${ }^{\dagger}$, to avoid collision. To increase operational availability, the approach procedure must accommodate a mixture of aircraft sizes and, consequently, approach speeds. The aircraft with the slower approach speed is placed in the lead position and the faster aircraft in the trail position. The fast aircraft first establishes dependent operation to the slow aircraft. The fast aircraft maintains speed and position against the slow aircraft until the fast aircraft reaches its final approach speed, which occurs after passing the final approach fix (FAF). For the remainder of the approach, the faster aircraft flies independently. It will close in on and, if the procedure allows, possibly pass the slower aircraft before landing. To appropriately position the fast aircraft before the FAF, it is necessary to determine the amount of separation that will be lost during the final approach.

The approach speeds of each aircraft and the approach geometry are the primary determinants of the separation loss during the approach. Given the approach geometry and speed schedule for each aircraft, one can use kinematics to compute the separation loss between a pair of aircraft. A kinematic model can complement Monte-Carlo simulations of the approach by enabling a tailored reduction in the variation of starting position for a given aircraft pair through identification of the ideal initial separation. The kinematic model could also be inserted into ground-based or on-board decision support tools to compute the optimal initial separation for a given pair of aircraft.

The precision of the kinematic model is limited by its assumptions. One challenge to improving the precision of a kinematic model is that aircraft do not fly the speed schedule using true airspeed. Aircraft use indicated or calibrated airspeed, which are functions of atmospheric properties at altitude. Therefore, even in segments of the descent where the scheduled speed is constant, the aircraft executes a slow deceleration during descent. Because the initial longitudinal separation of aircraft in a paired approach is less than a nautical mile, the impact of the slow deceleration on loss of separation, though small, is significant. Therefore, the kinematic model should capture this effect. Using the 1976 U.S. Atmosphere Model, the paper formulates a kinematic model using equivalent airspeed (EAS) to better approximate the indicated or calibrate airspeed flown by the aircraft. That EAS model mirrors a kinematic model that uses true airspeed by introducing an equivalent airspeed distance $\left(\mathrm{D}_{\mathrm{EAS}}\right)$. The predicted loss of separation using the EAS model is compared against a high-fidelity simulation performing the same approach.

\section{Approach Geometry}

Candidate geometries for closely spaced parallel approaches (CSPO) are detailed in references 1 through 6. To summarize, air traffic control vectors a pair of aircraft onto parallel approach paths at a point between the initial approach fix (IAF) and the final approach fix (FAF). The aircraft with the faster final approach speed is placed behind the aircraft with the slower final approach speed. The aircraft also begin with a vertical separation of $1000 \mathrm{ft}$ to allow each aircraft to safely establish their approach path and to allow the fast aircraft to establish longitudinal separation from the slow aircraft. The faster aircraft can start at either the higher altitude or the lower altitude. Each aircraft flies at a constant altitude until encountering the glidepath and then descends along the glidepath. From the start of the procedure to the FAF, the slow aircraft flies an assigned constant speed (nominally $180 \mathrm{KT}$ ) and the fast aircraft maintains speed and position against the slow aircraft. The fast aircraft executes this dependent operation until it reaches its final approach speed (matching speed of the slow aircraft as the slow aircraft decelerates upon reaching the FAF) or, if the fast aircraft does not detect the acceleration of the slow aircraft prior to the reaching the FAF, the fast aircraft switches to independent operation at the FAF.

${ }^{\dagger}$ An aircraft is said to blunder when it departs from its approach path toward the path of the other aircraft. A blunder may occur due to pilot error or aircraft malfunction. 


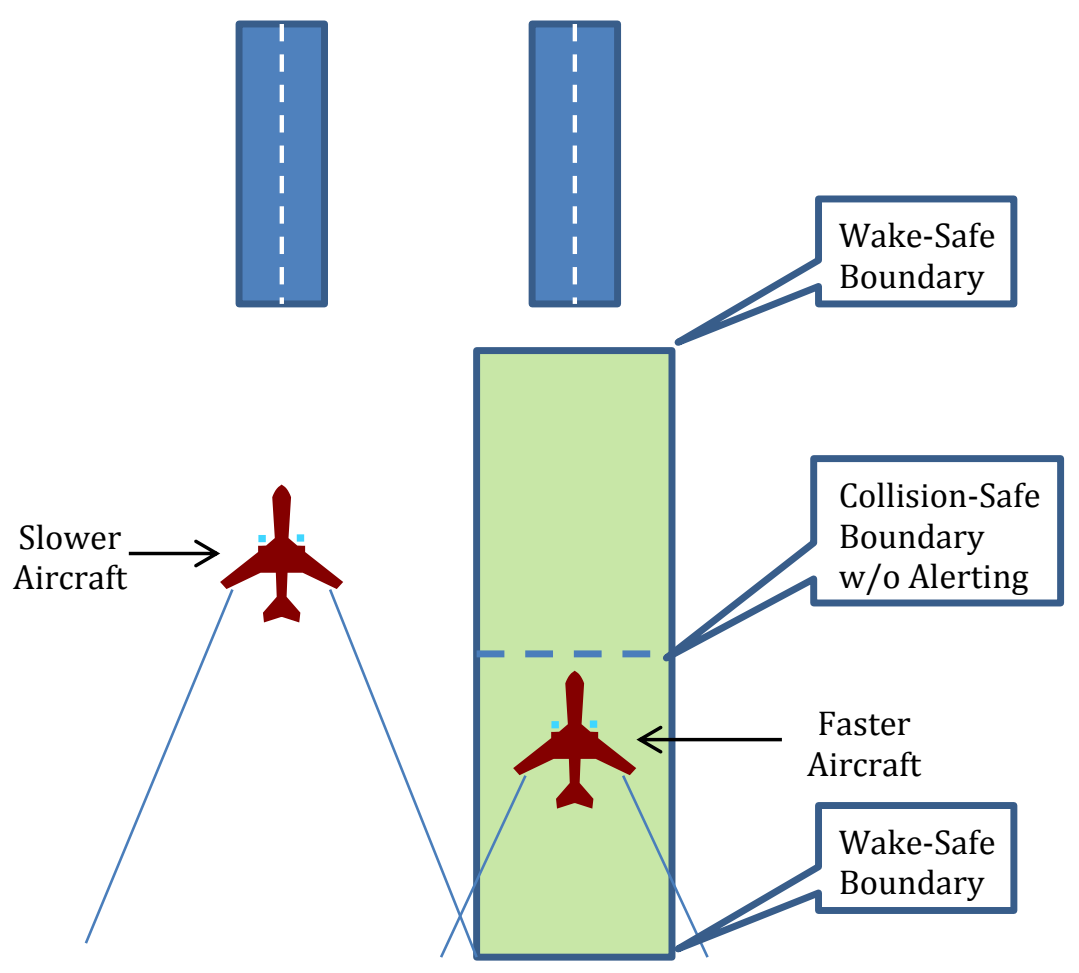

Figure 1: Paired Approach Separation Distances

Figure 1 depicts the horizontal geometry of the paired-approach procedure. The figure shows two options for the separation window (i.e. the green box), the full length of the box applies to a procedure that allows the fast aircraft to pass the slow aircraft ${ }^{1}$ and the hashed boundary applies to a procedure that does not allow the fast aircraft to pass ${ }^{6}$. The former requires the trail aircraft to be equipped with a collision alerting and avoidance system that is designed for the tight vertical and longitudinal separations in the paired approach. The latter is designed to be passively safe against collisions and, therefore, requires no special equipage. In each option, the separation window is defined to avoid two hazards. In the case where passing is not allowed, those hazards are collision and wake. To avoid collision, the fast aircraft must maintain a sufficient distance behind the slow aircraft such that the slow aircraft will not collide with the fast aircraft if the slow aircraft blunders across the fast aircraft's path. To avoid wake, the fast aircraft must be close enough to the slow aircraft that the wake of the slow aircraft passes behind the fast aircraft in the presence of adverse crosswind. In the case were passing is allowed, the two hazards are both wake encounters. The fast aircraft must remain forward of a rear wake-safe boundary to avoid encountering the wake of the slower aircraft. The fast aircraft must also remain behind a forward wake-safe boundary so that the slow aircraft does not encounter the wake of the fast aircraft. The paired approach with passing relies on collision alerting rather than separation to prevent collisions when one or both aircraft blender. With or without passing, the fast aircraft must maintain its separation between the forward and rear boundaries from the loss of altitude separation until one of the aircraft crosses the threshold.

The kinematic models derived in this paper assume that the runway thresholds are not offset but they can have different elevations. Section III.E.3 discusses adaptation of the models for offset runways. If the runway thresholds are offset, then the slow aircraft is assigned to the closer runway. From inspection, the kinematic models should be applicable to runways with different glideslope angles, but the models have not been verified and validated under this scenario. If a runway pair does have differential glideslopes, then the slow aircraft flies the shallower glideslope since wake vortices tend to descend.

${ }^{\ddagger}$ Both collision and wake risks are assumed to be negligible after the lead aircraft crosses the threshold. The assumption on collision risk is reasonable given that ground hazards restrict banking by the lead aircraft and the go-around procedure can be designed to increase lateral separation. However, further wake studies on landing and go around scenarios are needed to validate the assumption of negligible wake risk. 


\section{Modeling Travel Time and Separation Loss}

Prior to the FAF, the fast aircraft uses speed management to actively control its longitudinal separation with the slow aircraft. The fast aircraft stops active management of longitudinal separation when it reaches its final approach speed (by decelerating in concert with the slow aircraft after the slow aircraft reaches the FAF) or when it reaches the FAF (if it has not detected deceleration by the slow aircraft beforehand). For the remainder of the procedure, the longitudinal separation will decrease due to the difference in approach speeds. However, the fast aircraft continues to monitor the longitudinal separation and will execute a goaround if it falls outside the separation window. Therefore, to limit nuisance go-arounds, the fast aircraft must position itself to accommodate the longitudinal compression before the slow aircraft reaches the FAF.

If the aircraft accurately fly their planned approach speed to the threshold, then the longitudinal compression is characterized by the approach speeds of the two aircraft, the rate of deceleration, the minimum separation that must be maintained, the height to which minimum separation must be maintained, winds, and atmospheric density. Winds and atmospheric density come into play because the approach speed is an indicated (or calibrated) airspeed, not a true ground speed. For simplicity, the model uses equivalent airspeed to approximate indicated airspeed. Also, winds will be ignored for the moment and addressed later in the paper.

Accounting for atmospheric density with altitude is desirable. Assuming a 1976 standard atmosphere, the equivalent airspeed at an altitude of $1800 \mathrm{ft}$ is about $3 \%$ lower than the true ground speed. From a FAF at $1800 \mathrm{ft}$ altitude to a touchdown crossing height $(\mathrm{TCH})$ at $50 \mathrm{ft}$ atltitude, the elapsed time to threshold is about 2 seconds shorter for the scheduled speed as EAS than if one assumes the scheduled speed is TAS. The challenge, however, in computing compression distance using EAS is that the conversion of EAS to TAS (necessary to apply kinematic principles) changes with altitude as density changes with altitude.

\section{A. Model with TAS $=$ EAS Assumption (TAS Model)}

Before incorporating the EAS to TAS conversion, the kinematic equations for the approach procedures are first formulated assuming the speed schedule is TAS (TAS model). Later, this paper will show how to formulate the EAS to TAS conversion in a way that resembles the TAS model. The speed schedule for the paired approach is depicted in Figure 1. The slow aircraft's speed schedule can be broken into three segments: a constant speed segment, a deceleration segment, and a final approach segment. The constant speed segment begins when the slow aircraft captures the glideslope and ends at the final approach fix. In this region, the aircraft flies a constant airspeed assigned by air traffic control. When the slow aircraft reaches the FAF, it begins the deceleration segment where it decelerates to its final approach speed. The model assumes a constant deceleration that achieves the final approach speed at the stabilized approach point (SAP), i.e. $1000 \mathrm{ft}$ AGL. The final approach segment continues after the SAP; the slow aircraft flies its final approach speed to the runway threshold. Separation monitoring ends when one of the two aircraft reaches the threshold so there is no need to model the flare maneuver after crossing the threshold.

To compute the compression distance at the FAF, one must first calculate the time it takes the slow aircraft to travel from the FAF to the end of the paired approach. If the fast aircraft is not allowed to pass, then the paired approach ends for the slow aircraft at the threshold. If the fast aircraft is allowed to pass, then the paired approach ends when the fast aircraft crosses the threshold. To avoid the wake of the fast aircraft, the slow aircraft must be behind by no more than the wake-safe distance $\left(\mathrm{D}_{\mathrm{wS}}\right)$. The location of the slow aircraft at the end of the paired approach will be called the procedure termination location for the slow aircraft or PTS.

The flight time from FAF to PTS is divided into two computations: the time from FAF to SAP and the time from SAP

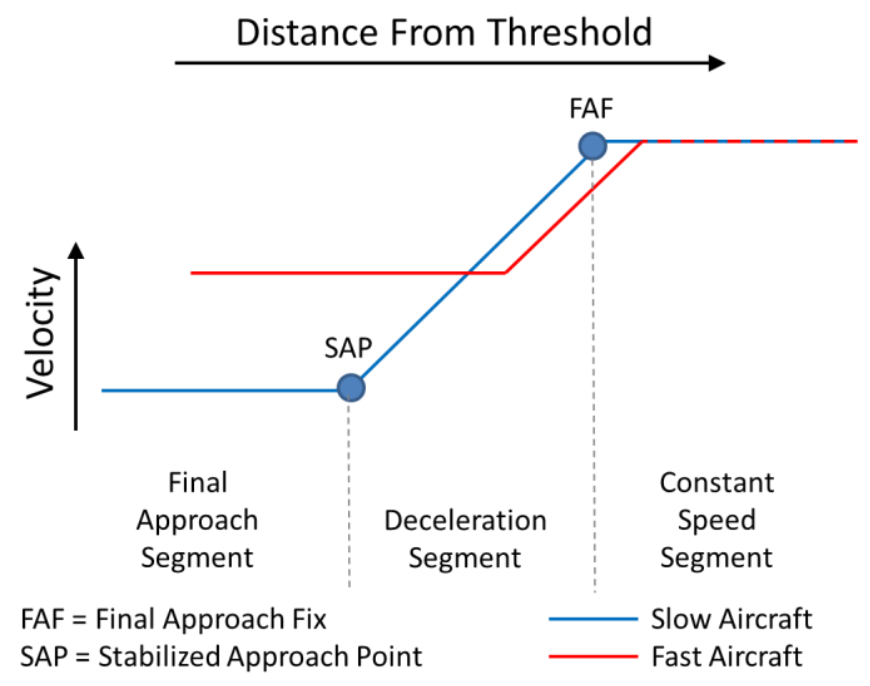

Figure 1. Speed Profiles of Slow and Fast Aircraft 
to PTS. The time from FAF to SAP assumes a constant deceleration from the constant segment speed $\left(\mathrm{V}_{\mathrm{c}}\right)$ to the final approach speed $\left(V_{f}\right)$. Thus, the average speed from FAF to SAP is $1 / 2\left[V_{c}+V_{f}\right]$. Given that the FAF and SAP are defined by height above the runway, the distance between these points is a function of their altitudes and the glideslope angle:

$$
t_{F A F \rightarrow S A P}=\frac{D(h, \gamma)}{V_{a v g}}=\frac{\left(h_{F A F}-h_{S A P}\right)}{\sin \gamma} \frac{2}{\left(V_{c}+V_{f}\right)} \frac{K T}{f p s}=\frac{18113}{\left(V_{c}+V_{f}\right)}
$$

where,

$\mathrm{D}(\mathrm{h}, \gamma)$ is the distance traveled as a function of altitude and glideslope angle

$\mathrm{V}_{\text {avg }} \quad$ is the average true speed of the aircraft

$\mathrm{h}_{\mathrm{FAF}} \quad$ is the geometric altitude of the FAF in feet, normally $1800 \mathrm{ft}$ above runway elevation

$\mathrm{h}_{\mathrm{SAP}} \quad$ is the geometric altitude of the SAP in feet, normally $1000 \mathrm{ft}$ above runway elevation

$\gamma \quad$ is the glideslope angle, normally $3^{\circ}$

$\mathrm{V}_{\mathrm{c}} \quad$ is the constant segment speed in knots, nominally $180 \mathrm{KT}$

$\mathrm{V}_{\mathrm{f}} \quad$ is the final approach speed in knots

$\mathrm{KT} / \mathrm{fps}$ is the conversion from knots to feet per second, 0.5924838

$\mathrm{t}_{\mathrm{FAF} \rightarrow \mathrm{SAP}}$ is the flight time from the FAF to the SAP in seconds

Similarly, the time from SAP to PTS is given by:

$$
t_{S A P \rightarrow P T S}=\frac{D(h, \gamma)}{V_{f}}=\frac{\left(h_{S A P}-h_{P T S}\right)}{V_{f} \sin \gamma} \frac{K T}{f p s}
$$

where, $h_{\text {PTS }}$ is the altitude of the procedure termination location for the slow aircraft:

$$
h_{P T S}=h_{T C H}+D_{W S} \tan \gamma
$$

In Eq. (3),

$\mathrm{D}_{\mathrm{WS}} \quad$ is the wake-safe distance. If passing is not allowed, this variable is zero.

$\mathrm{h}_{\mathrm{TCH}} \quad$ is the altitude of threshold crossing height, nominally $50 \mathrm{ft}$ above runway elevation

In the example of a paired approach without passing, $\mathrm{D}_{\mathrm{WS}}$ is zero and $\mathrm{h}_{\mathrm{PTS}}$ equals $\mathrm{h}_{\mathrm{TCH}}$. Given a generic runway at sea-level elevation with a TCH of $50 \mathrm{ft}$ AGL, Eq. (2) reduces to:

$$
t_{S A P \rightarrow P T S}=t_{S A P \rightarrow T C H}=\frac{\left(h_{S A P}-h_{T C H}\right)}{V_{f} \sin \gamma} \frac{K T}{f p s}=\frac{10755}{V_{f}}
$$

Then, the total flight time from FAF to TCH for the slow aircraft landing at the generic runway is:

$$
t_{\text {slow }}=t_{F A F \rightarrow S A P}+t_{S A P \rightarrow P T S}=\frac{18113}{\left(V_{c}+V_{f}\right)}+\frac{10755}{V_{f}}
$$

For example, if $\mathrm{V}_{\mathrm{c}}$ is $180 \mathrm{KT}$ and $\mathrm{V}_{\mathrm{f}}$ is $120 \mathrm{KT}$, then the $\mathrm{t}_{\mathrm{slow}}$ is 150.0 seconds.

Next, one calculates where the fast aircraft would be $t_{\text {slow }}$ seconds in the past. This calculation depends on the separation that the fast aircraft must maintain relative to the slow aircraft at the runway threshold. In the paired procedure without passing, the faster aircraft must maintain a collision free distance $\left(\mathrm{D}_{\mathrm{CS}}\right)$ from slower aircraft upon procedure termination. For the paired approach with passing, the procedure terminates for the fast aircraft at the threshold. As with the slow aircraft, this required separation is converted into a procedure termination altitude for the faster aircraft, $\mathrm{h}_{\mathrm{PTF}}$ :

$$
h_{P T F}=h_{T C H}+D_{C S} \tan \gamma
$$

where,
$\mathrm{D}_{\mathrm{CS}}$
is the collision-safe distance. If passing is allowed, this variable is zero.
$\mathrm{h}_{\mathrm{TCH}}$ is the altitude of the threshold crossing height, nominally $50 \mathrm{ft}$ above runway elevation
$\gamma \quad$ is the glideslope angle, nominally $3^{\circ}$ 
Note that the $\mathrm{h}_{\mathrm{TCH}}$ in Eq. (6) can differ from $\mathrm{h}_{\mathrm{TCH}}$ in Eq. (3) to accommodate runways with different threshold crossing heights; moreover, because the geometric altitude and not the runway relative altitude is used, the runways can also have different elevations. Similarly, for Eq. (7) to Eq. (15) that follow, $\mathrm{h}_{\mathrm{SAP}}$ and $\mathrm{h}_{\mathrm{FAF}}$ can differ for the fast aircraft to accommodate different runway elevations or approach geometries. To handle differential glideslopes, the glideslope angle in Eq. (7) to Eq. (15) can also differ from that in Eq. (3) except where noted.

One aspect of the paired procedure that complicates the computation is the condition on the time and deceleration for the fast aircraft. The fast aircraft decelerates at the same rate as the slow aircraft when and if it detects the deceleration of the slow aircraft prior to the fast aircraft reaching the FAF; otherwise, the fast aircraft decelerates independently. The parameter governing this condition is $t_{\text {delay }}$, the time delay in the response of the fast aircraft to changes in the sensed state of the slow aircraft. This exercise will assume that the speed of the slow aircraft is monitored using ADS-B and exhibits a $t_{\text {delay }}$ of 5.0 seconds. Table 1 provides a breakdown of the estimated $t_{\text {delay }}$.

Table 1. Estimate of Response Delay in Dependent Operations

\begin{tabular}{|l|l|}
\hline $\begin{array}{l}\text { ADS-B OUT Latency (from } \\
\text { navigation measurement) }\end{array}$ & $2.0 \mathrm{~s}$ \\
\hline ADS-B IN Latency & $0.5 \mathrm{~s}$ \\
\hline Speed Guidance Algorithm & $0.5 \mathrm{~s}$ \\
\hline Pilot Reaction Time & $2.0 \mathrm{~s}$ \\
\hline Total & $5.0 \mathrm{~s}$ \\
\hline
\end{tabular}

Let $t_{\text {I-fast }}$ be the independent flight time of the fast aircraft from the FAF to PTF. The fast aircraft will decelerate independently if $\left(t_{\text {slow }}-t_{\text {I-fast }}\right)<t_{\text {delay }}$. The equations for $t_{\text {I-fast }}$ are identical to those for $t_{\text {slow }}$ but $\mathrm{h}_{\mathrm{PTF}}$ is substituted for $\mathrm{h}_{\mathrm{PTS}}$. For this example using the generic runway, the collision-safe distance, $\mathrm{D}_{\mathrm{CS}}$, is set to $750 \mathrm{ft}$.

$$
\begin{gathered}
h_{P T F}=h_{T C H}+D_{C S}(\tan \gamma)=50 f t+750 f t\left(\tan 3^{\circ}\right)=89.3 f t \\
t_{S A P \rightarrow P T F}=\frac{D(h, \gamma)}{V}=\frac{\left(h_{S A P}-h_{P T F}\right)}{V_{f} \sin \gamma} \frac{K T}{f p s}=\frac{10310}{V_{f}} \\
t_{I-f a s t}=t_{F A F \rightarrow S A P}+t_{S A P \rightarrow P T F}=\frac{18113}{\left(V_{c}+V_{f}\right)}+\frac{10310}{V_{f}}
\end{gathered}
$$

If the value of $t_{\text {I-fast }}$ reveals that the fast aircraft will perform an independent deceleration, then the position of the $\mathrm{x}$-axis runway coordinate of the fast aircraft when the slow is at the FAF is:

$$
X_{I-\text { fast }}=X_{F A F}-\cos \gamma\left(V_{c}\left(t_{\text {slow }}-t_{I-\text { fast }}\right) \frac{f p s}{K T}\right)
$$

where $\mathrm{X}_{\mathrm{FAF}}$ is the $\mathrm{x}$-axis runway coordinate of the $\mathrm{FAF}$, nominally $-33,392 \mathrm{ft}$ or $-5.5 \mathrm{NM}$. If $\mathrm{X}_{\mathrm{FAF}}$ differs between the two runways, then use $\mathrm{X}_{\mathrm{FAF}}$ for the runway of the fast aircraft in Eq. (10).

If the fast aircraft will perform a dependent deceleration with the slow aircraft, the fast aircraft's total flight time, $t_{D-f a s t}$, can be broken into three components: 1$) t_{\text {delay }}$ - the delay in dependent operation defined in the previous paragraph, 2) $t_{\text {decel }}$ - the time to decelerate to the final approach speed, and 3) $t_{\text {final }}$ - the time flying at the final approach speed. $t_{\text {decel }}$ is computed using the deceleration of the slow aircraft (due to the dependent operation) and the final approach speed of the fast aircraft:

$$
a_{\text {slow }}=\left(\frac{\Delta V}{t_{F A F \rightarrow S A P}}\right)_{\text {slow }}=\left(V_{f}-V_{c}\right) \frac{\left(V_{c}+V_{f}\right)}{2} \frac{\sin \gamma}{\left(h_{F A F}-h_{S A P}\right)} \frac{f p s}{K T}=\frac{\left(V_{f}^{2}-V_{c}^{2}\right)}{18113}
$$




$$
t_{\text {decel }}=\frac{\Delta V_{\text {fast }}}{a_{\text {slow }}}=18113 \frac{\left(V_{f}-V_{c}\right)_{\text {fast }}}{\left(V_{f}^{2}-V_{c}^{2}\right)_{\text {slow }}}
$$

Note that the equation for $\mathrm{a}_{\text {slow }}$ produces deceleration in units of $\mathrm{kt} / \mathrm{s}$ so that the equation for $\mathrm{t}_{\text {decel }}$ produces a time in units of seconds. Also, note that in the case of differential glideslopes, Eq. (11) uses the glideslope angle for the slow aircraft. Now, $\mathrm{t}_{\text {final }}$ becomes the remaining time:

$$
t_{\text {final }}=t_{\text {slow }}-t_{\text {delay }}-t_{\text {decel }}
$$

Next, compute the runway $\mathrm{x}$-axis location of the fast aircraft performing a dependent deceleration:

$$
X_{D-f a s t}=-\left(\mathrm{D}_{C S}+\cos \gamma\left(V_{f} t_{\text {final }}+\frac{1}{2}\left(V_{c}+V_{f}\right) t_{\text {decel }}+V_{c} t_{\text {delay }}\right) \frac{f p S}{K T}\right)
$$

Now, compute the required longitudinal separation at the FAF for the aircraft pair:

$$
\mathrm{S}_{F A F}=X_{F A F}-\left\{\begin{array}{l}
X_{I-\text { fast }} \text { if }\left(t_{\text {slow }}-\mathrm{t}_{\mathrm{I}-\text { fast }}\right)<\mathrm{t}_{\text {delay }} \\
X_{D \text {-fast }} \text { if }\left(t_{\text {slow }}-\mathrm{t}_{\mathrm{I} \text {-fast }}\right) \geq \mathrm{t}_{\text {delay }}
\end{array}\right.
$$

If $\mathrm{X}_{\mathrm{FAF}}$ differs between runways, then use $\mathrm{X}_{\mathrm{FAF}}$ for the runway of the slow aircraft in Eq. (15). Equation (15) assumes that the runway thresholds are not offset in the $\mathrm{X}$-axis direction.

The compression distance is the difference between longitudinal separation at the FAF and longitudinal separation at the threshold:

$$
d_{\text {compress }}=S_{F A F}-D_{C S}+D_{W S}
$$

(Recall that $\mathrm{D}_{\mathrm{CS}}$ is zero for the paired approach with passing and that $\mathrm{D}_{\mathrm{WS}}$ is zero for the paired approach without passing. So, at procedure termination, the fast aircraft is either a distance of $\mathrm{D}_{\mathrm{CS}}$ behind the slow aircraft or a distance of $D_{\mathrm{ws}}$ ahead of the slow aircraft.)

Continuing the example, if one computes the compression distance for a paired approach without passing where the slow aircraft final approach speed is $120 \mathrm{KT}$, the fast aircraft final approach speed is 130 $\mathrm{KT}$, the constant segment speed is $180 \mathrm{KT}$, and the collision safe distance $\mathrm{D}_{\mathrm{CS}}$ is $750 \mathrm{ft}$, then the results are:

$$
\begin{array}{ll}
=150.0 \text { seconds } \\
\mathrm{t}_{\text {slow }} & =137.7 \text { seconds, }\left(\mathrm{t}_{\text {slow }}-\mathrm{t}_{\mathrm{I} \text {-fast }}\right)=12.3 \text { seconds }>\mathrm{t}_{\text {delay }} \text { so compute } \mathrm{X}_{\mathrm{D} \text {-fast }} \\
\mathrm{t}_{\mathrm{I} \text {-fast }} & =50.3 \text { seconds } \\
\mathrm{t}_{\text {decel }} & =-36159 \mathrm{ft} \\
\mathrm{X}_{\mathrm{D} \text {-fast }} & =2767 \mathrm{ft} \\
\mathrm{S}_{\mathrm{FAF}} & =2017 \mathrm{ft} \\
\mathrm{d}_{\text {compress }} &
\end{array}
$$

\section{B. Including the EAS to TAS Conversion (EAS Model)}

Next, one incorporates the EAS to TAS conversion to fly the speed schedule as EAS:

$$
V_{T A S}=V_{E A S} \sqrt{\frac{\rho_{0}}{\rho}}=V_{E A S} \sqrt{\frac{P_{0} T}{P T_{0}}}
$$

where $\rho, \mathrm{P}$, and $\mathrm{T}$ are the atmospheric density, pressure, and temperature respectively and the subscript zero indicates the sea-level value. The second formulation allows the insertion of functions for temperature and pressure from the US Standard 1976 atmosphere model for altitudes below $36,152 \mathrm{ft}:{ }^{8}$

$$
H=\frac{h R_{e}}{h+R_{e}}
$$




$$
\begin{gathered}
T=T_{0}\left(1+\frac{H L}{T_{0}}\right) \\
P=P_{0}\left(1+\frac{H L}{T_{0}}\right)^{-g /(L R)}
\end{gathered}
$$

where,

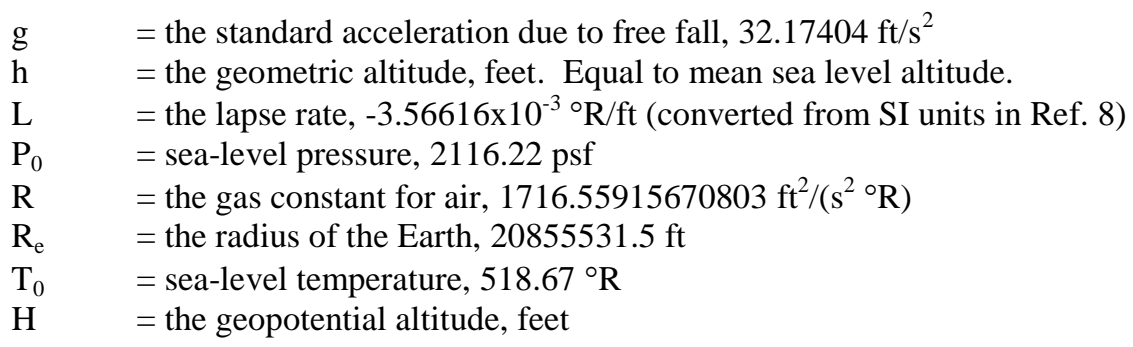

With these equations, the conversion from EAS to TAS becomes:

$$
\begin{aligned}
V_{T A S}=V_{E A S} f(h) & =V_{E A S} \sqrt{\left(1+\frac{h L R_{e}}{\left(h+R_{e}\right) T_{0}}\right)^{1+g /(L R)}} \\
& =V_{E A S} \sqrt{\left(1-\frac{143.394 h}{h+20855531.5}\right)^{-4.25589}}
\end{aligned}
$$

Equation (21) computes TAS as a function of geometric altitude, h. To determine the distance traveled over a given time, we recognize that TAS is $\mathrm{dr} / \mathrm{dt}$ where $\mathrm{r}$ is the distance along the glideslope. Since the glideslope angle is constant, we can convert $\mathrm{dr} / \mathrm{dt}$ to $\mathrm{dh} / \mathrm{dt}$ using the relation, $\mathrm{h}=\mathrm{r} \sin (\gamma)$.

$$
\begin{gathered}
V_{T A S}=\left(\frac{K T}{f p s}\right) \frac{d r}{d t}=\left(\csc \gamma \frac{K T}{f p s}\right) \frac{d h}{d t}=V_{E A S} f(h) \\
\int d t=t=\left(\frac{\csc \gamma}{V_{E A S}} \frac{K T}{f p s}\right) \int \frac{1}{f(h)} d h
\end{gathered}
$$

The integral for dh does not have a closed form solution. However, one can construct a closed form approximation by converting the integrand to a Taylor series and integrating the first two terms:

$$
\begin{gathered}
t=\left(\frac{\csc \gamma}{V_{E A S}} \frac{K T}{f p s}\right) \int\left(1-1.46309 \times 10^{-5} h+5.74347 \times 10^{-11} h^{2}\right) d h \\
=\frac{\csc \gamma\left(h-7.31543 \times 10^{-6} h^{2}+1.91449 \times 10^{-11} h^{3}\right)}{V_{E A S}} \frac{K T}{f p s}=\frac{D_{E A S}(h)}{V_{E A S}} \frac{K T}{f p s}
\end{gathered}
$$

As indicated by its final formulation, Eq. (24) defines an equivalent airspeed distance ( $\left.D_{\text {EAS }}\right)$ that is a function of geometric altitude. Note that removing the second and third order terms reduces Eq. (24) to the assumption that TAS $=$ EAS. Thus, the second and third order terms represent the effect of the EAS $\rightarrow$ TAS conversion on the time to reach sea level. For example, when starting at a $1000 \mathrm{ft}$ altitude, the higher order terms indicate that the EAS $\rightarrow$ TAS conversion is equivalent to reducing the distance traveled by $139 \mathrm{ft}$. The third order term produces a distance of less than 1 foot below $\mathrm{h}=3738 \mathrm{ft}$. It can be ignored for runways at elevations below $1500 \mathrm{ft}$. 
We can substitute $D_{\text {EAS }}$ for the $h / \sin (\gamma)$ terms in Eq. (1) through Eq. (12). For example, the time to travel from SAP to PTS, Eq. (1), becomes:

$$
t_{S A P \rightarrow P T S}=\frac{D(h, \gamma)}{V_{f}}=\frac{\left(D_{E A S}\left(h_{S A P}\right)-D_{E A S}\left(h_{P T S}\right)\right)}{V_{f}} \frac{K T}{f p S}
$$

This substitution also works for the deceleration segment when deceleration is a constant deceleration of the equivalent airspeed because a constant deceleration term can be evaluated in the time integral:

$$
\begin{gathered}
V_{T A S}=\left(V_{E A S}^{0}+a_{E A S} t\right) f(h)=\left(\csc \gamma \frac{K T}{f p S}\right) \frac{d h}{d t} \\
\int\left(V_{E A S}^{0}+a_{E A S} t\right) \frac{f p s}{K T} d t=\csc \gamma \int \frac{1}{f(h)} d h \\
\left(V_{E A S}^{0} t+\frac{1}{2} a_{E A S} t^{2}\right) \frac{f p s}{K T}=D_{E A S}(h)
\end{gathered}
$$

Equation (28) is identical to the true airspeed kinematic equation for constant acceleration, with $\mathrm{D}_{\mathrm{EAS}}$ replacing the distance term. Finally, Eq. (1) through Eq. (12) are modified to use $D_{\text {EAS. }}$ Using example values for the generic runway, the final equations for a paired approach without passing are:

$$
\begin{gathered}
t_{\text {slow }}=\left(\frac{\left(\mathrm{D}_{\mathrm{EAS}}(1000)-\mathrm{D}_{\mathrm{EAS}}(50)\right)}{V_{f}}+\frac{2\left(\mathrm{D}_{\mathrm{EAS}}(1800)-\mathrm{D}_{\mathrm{EAS}}(1000)\right)}{V_{f}+V_{c}}\right) \frac{K T}{f p s} \\
=\frac{10672}{V_{f}}+\frac{17744}{V_{f}+V_{c}} \\
t_{I-\text { fast }}=\frac{\left(\mathrm{D}_{\mathrm{EAS}}(1000)-\mathrm{D}_{\mathrm{EAS}}\left(50+\mathrm{D}_{C S} \tan 3^{\circ}\right)\right)}{V_{f}} \frac{K T}{f p s}+\frac{17744}{V_{f}+V_{c}}=\frac{10228}{V_{f}}+\frac{17744}{V_{f}+V_{c}} \\
a_{\text {slow }}=\left(\frac{\Delta V}{t_{\text {FAF } \rightarrow \text { SAP }}}\right)_{\text {slow }}=\left(V_{f}-V_{c}\right) \frac{V_{f}+V_{c}}{17744}=\frac{\left(V_{f}^{2}-V_{c}^{2}\right)}{17744} \\
t_{\text {decel }}=\frac{\Delta V_{\text {fast }}}{a_{\text {slow }}}=17744 \frac{\left(V_{f}-V_{c}\right)_{\text {fast }}}{\left(V_{f}^{2}-V_{c}^{2}\right)_{\text {slow }}}
\end{gathered}
$$

To compute the runway $x$-axis coordinate of the fast aircraft when the slow aircraft is at the FAF, one first computes the position as a $\mathrm{D}_{\mathrm{EAS}}$ distance:

$$
\begin{gathered}
D_{I-\text { fast }}=D_{E A S}\left(h_{F A F}\right)+V_{c}\left(t_{\text {slow }}-t_{I-\text { fast }}\right) \frac{f p s}{K T} \\
D_{D-\text { fast }}=\mathrm{D}_{\mathrm{EAS}}\left(h_{P T F}\right)+\left(V_{f} t_{\text {final }}+\frac{1}{2}\left(V_{c}+V_{f}\right) t_{\text {decel }}+V_{c} t_{\text {delay }}\right) \frac{f p s}{K T} \\
=1705+\left(V_{f} t_{\text {final }}+\frac{1}{2}\left(V_{c}+V_{f}\right) t_{\text {decel }}+V_{c} t_{\text {delay }}\right) \frac{f p s}{K T} \\
D_{\text {fast }}=\left\{\begin{array}{l}
D_{\text {I-fast }} \text { if }\left(t_{\text {slow }}-\mathrm{t}_{\text {I-fast }}\right) \leq \mathrm{t}_{\text {delay }} \\
D_{D-\text { fast }} \text { if }\left(t_{\text {slow }}-\mathrm{t}_{\text {I-fast }}\right)>\mathrm{t}_{\text {delay }}
\end{array}\right.
\end{gathered}
$$

Then, solve the $\mathrm{D}_{\mathrm{EAS}}$ formula to determine the starting geometric altitude: 


$$
\begin{aligned}
& \csc \gamma\left(h-7.31543 \times 10^{-6} h^{2}+1.91449 \times 10^{-11} h^{3}\right)=D_{\text {fast }} \\
& 1.91449 \times 10^{-11} h^{3}-7.31543 \times 10^{-6} h^{2}+h-D_{\text {fast }} \sin \gamma=0
\end{aligned}
$$

The last term, $\mathrm{D}_{\text {fast }} \sin \gamma$, represents an equivalent airspeed altitude, $\mathrm{h}$. Thus, the resulting solution will be a conversion from the equivalent airspeed altitude (h') to the geometric altitude (h). The real cube root of Eq. (37) is a complicated formula. To simplify it, the cube root is expanded into a Taylor series and the first four terms of the series are used:

$$
h_{\text {fast }}=h^{\prime}+7.31543 \times 10^{-6} h^{\prime 2}+8.78862 \times 10^{-11} h^{\prime 3}+1.25718 \times 10^{-15} h^{\prime 4}
$$

The runway $\mathrm{x}$-axis coordinate for the fast aircraft is then:

$$
X_{\text {fast }}=-\cot \gamma\left(h_{\text {fast }}-h_{T C H}\right)
$$

As with the TAS model, different values of $\mathrm{h}_{\mathrm{TCH}}, \mathrm{h}_{\mathrm{FAF}}$, and $\gamma$ can be used for the fast aircraft in Eq. (6), the $D_{\text {EAS }}$ equivalent of Eq. (8), and Eq. (33) to Eq. (39) to accommodate runways with different threshold crossing heights, different elevations, or different glideslope angles.

Finally, the required longitudinal separate at the FAF is:

$$
S_{F A F}=X_{F A F}-X_{\text {fast }}
$$

If $\mathrm{X}_{\mathrm{FAF}}$ differs between the two runways, then use $\mathrm{X}_{\mathrm{FAF}}$ for the runway of the slow aircraft. Equation (40), like Eq. (15), assumes that the runway thresholds are not offset along the x-runway axis direction. To compute the separation compression use Eq. (16).

Using the prior example of the slow aircraft with a final approach speed of $120 \mathrm{KT}$ and a fast aircraft with a final approach speed of $130 \mathrm{KT}$, the results using the EAS model are:

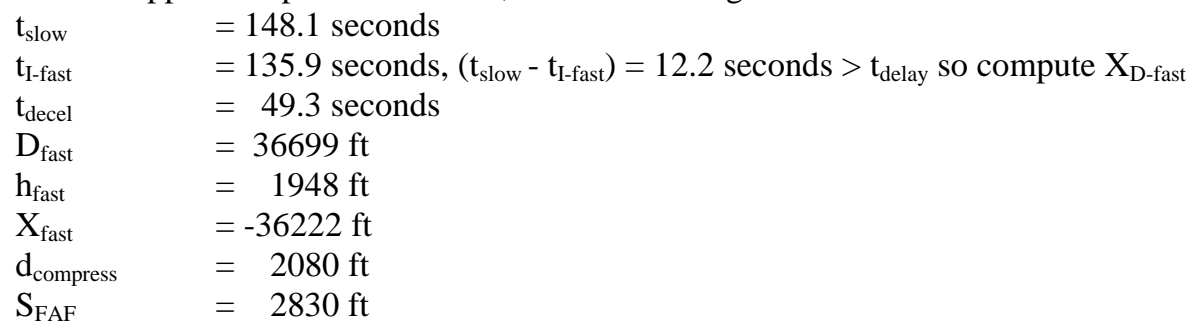

\section{Comparison of Kinematic Models}

In comparison to the earlier example using the TAS model, the compression length increased from 2017 $\mathrm{ft}$ to $2080 \mathrm{ft}$, a difference of $63 \mathrm{ft}$. So, how can the compression length be larger when the time of flight for both aircraft is shorter? The answer is that the fast aircraft maintains a higher altitude and, therefore, has a slightly higher true airspeed, even when the equivalent airspeed is equal to the slow aircraft. Depending on the initial separation, this effect can cause added compression that is greater than the reduced compression from the shorter flight time. It is one limitation of the EAS model. In the procedure, the fast aircraft uses a speed management algorithm that maintains separation through deceleration, effectively causing the fast aircraft to track the true airspeed of the slow aircraft. Therefore, until the fast aircraft decelerates to its final approach speed as an equivalent airspeed, the fast aircraft's equivalent airspeed should be slightly lower than the equivalent airspeed of the slow. One could partly compensate for this effect by giving the fast aircraft a slightly lower EAS for the constant segment.

The above example places the fast aircraft about $148 \mathrm{ft}$ above the slow aircraft when the slow aircraft reaches the FAF. If the slow aircraft has an EAS of $180 \mathrm{KT}$ and the fast aircraft is matching the true airspeed of the slow aircraft, then the EAS of the fast aircraft would be 179.6 KT when the slow aircraft is at the FAF. Using this slightly lower EAS for $\mathrm{V}_{\mathrm{c}}$ for the fast aircraft would reduce the compression distance to $2042 \mathrm{ft}$, a change of $38 \mathrm{ft}$ that leaves a difference between the two models of $25 \mathrm{ft}$. This adjustment does, however, require at least two iterations of Eq. (29) through Eq. (40). The first iteration approximates $\mathrm{h}_{\text {fast }}$ in order to adjust $\mathrm{V}_{\mathrm{c}}$ for the fast aircraft using Eq. (21), and the second iteration 
substitutes the adjusted $\mathrm{V}_{\mathrm{c}}$. Otherwise, there is no simple mechanism to modify the EAS-based model to emulate true airspeed matching through deceleration. Nevertheless, the example results do demonstrate that the EAS-based model often provides the more conservative estimate of separation compression. Moreover, the EAS-based model remains sufficiently simple to program or to use in generating look-up tables; therefore, it can be used in real-time by flight deck or controller systems to generate custom values of the front-gate separation for specific aircraft pairs.

The differences between the EAS kinematic model and the TAS kinematic model are very modest in the sea-level example. The difference in travel time does change substantially with runway elevation. Changing runway elevation to $1000 \mathrm{ft}$ in the example above decreases $t_{\text {slow }}$ by 4.1 seconds compared to the TAS model, almost double the difference at sea level. However, the difference in separation compression is less sensitive to elevation since compression is a function of relative velocity between the aircraft pairs and the relative velocity changes more slowly with elevation given that each aircraft experiences nearly the same EAS-to-TAS conversion along the path. The difference in separation compression grows to only 70 $\mathrm{ft}$ or, if setting $\mathrm{V}_{\mathrm{c}}$ of the fast aircraft to $179.6 \mathrm{KT}$ to match the true airspeed of the slow aircraft at the FAF, the compression difference grows to $32 \mathrm{ft}$.

The compression difference, however, is more sensitive to the difference in approach speed between the slow and fast aircraft. If the fast aircraft speed were increased to $140 \mathrm{KT}$ on approach to the generic runway, then the difference in separation compression increases to 96 feet or, if setting $\mathrm{V}_{\mathrm{c}}$ of the fast aircraft to $179.6 \mathrm{KT}$, the difference increases to $65 \mathrm{ft}$. Thus, the EAS model becomes more relevant when analyzing the limit on the speed differential for a paired approach procedure. Also, as paired approaches are applied to runway separations as little as 700 feet, wake considerations drive the separation window to smaller lengths and require ever tighter performance margins. Ref. 4 estimated that the fast aircraft must be able to maintain position within a separation window of 490 feet to enable parallel, paired approaches without passing at $750 \mathrm{ft}$ runway separation. The differences in separation compression estimates, therefore, become a greater percentage of the separation window as runway separation decreases and can influence the determination of required aircraft navigation performance and feasible aircraft pairings.

\section{Comparison of Kinematic Models to High Fidelity Simulation}

To validate the EAS model, its predictions of travel time from FAF to runway threshold were compared against a high fidelity simulation of a large civil transport. The high-fidelity simulation is one of the models available in the Cockpit Motion Facility at NASA Langley Research Center. ${ }^{9}$ The high-fidelity simulation executed the approach using an autothrottle programmed to the speed schedule and an autopilot configured to follow localizer and glideslope signals. Furthermore, sensor errors were disabled so that the modeled aircraft would better conform to the approach path. However, conforming to the speed schedule was more challenging. The high fidelity simulation doesn't have an autothrottle mode to mimic the constant deceleration in the kinematic models. Moreover, deceleration depends in part on landing gear deployment, flaps settings, and speed brake settings, all of which require pilot action. Therefore, the simulation uses a pseudo-pilot algorithm to operate the levers for gear, flaps, and speed brake. In this simulation, the speed-brake deployment is a one-time action at deceleration start, and the lever position is a function of the the target deceleration. The speed brake is then retracted prior to achieving the final approach speed. Table 2 shows the predictions of travel time using the TAS model, the EAS model, and the simulation. The approach was to KSFO RWY28L $(\mathrm{TCH}=57 \mathrm{ft}$, runway elevation $=13 \mathrm{ft}$ ):

Table 2. Comparison of Approach Times.

\begin{tabular}{|c|c|r|r|}
\hline \multirow{2}{*}{$\begin{array}{c}\text { Approach } \\
\text { Speed (KT) }\end{array}$} & \multicolumn{3}{|c|}{ Time from FAF to TCH (seconds) } \\
\cline { 2 - 4 } & model & EAS Model & $\begin{array}{c}\text { High } \\
\text { Fidelity } \\
\text { Simulation }\end{array}$ \\
\hline 120 & 149.34 & 147.58 & 146.66 \\
\hline 130 & 140.55 & 138.87 & 138.58 \\
\hline 140 & 132.86 & 131.25 & 131.34 \\
\hline 150 & 126.06 & 124.52 & 124.84 \\
\hline 160 & 120.00 & 118.52 & 118.12 \\
\hline
\end{tabular}


Except for the $120 \mathrm{KT}$ approach speed, the estimates of travel time from the FAF to the runway threshold are nearly the same (within \pm 0.4 seconds) for the EAS model and the high fidelity simulation. For the $120 \mathrm{KT}$ approach speed, the high fidelity simulation arrives 0.92 seconds earlier than estimated by the EAS model. The $120 \mathrm{KT}$ approach speed requires a deceleration near the limit of what the modeled aircraft can achieve when autothrottle is engaged, and the actual speed of the aircraft in the high fidelity simulation is closer to $121 \mathrm{KT}$ at the SAP. When the EAS kinematic model was re-evaluated with an approach speed of $121 \mathrm{KT}$, then the estimated time to threshold changed to 146.47 seconds, which is a closer match to the high fidelity simulation.

\section{E. Variations to the EAS Kinematic Model}

\section{Deviations from Standard Day Density}

The EAS model, as presented in section III.B, is tailored to the standard day profile for density in the 1976 Atmosphere Model. Actual density that departs from the standard day profile will also adjust the compression distance. One can employ the methods used to generate Eq. (24) against adjustments to the standard day. For example, when adjusting density by changing the sea-level temperature, the coefficients of Eq. (24) can be formulated as functions of the sea-level temperature $\left(\mathrm{T}_{0}\right)$ in degrees Rankine:

$$
\begin{aligned}
D_{E A S}\left(h, T_{0}\right)= & \csc \gamma\left(h-\frac{0.00379429}{T_{0}} h^{2}\right. \\
& \left.+\frac{\left(5.08743 \times 10^{-6}+1.21288 \times 10^{-11} \mathrm{~T}_{0}\right)}{T_{0}^{2}} h^{3}\right)
\end{aligned}
$$

Unfortunately, the conversion back to geometric altitude, i.e. the real root of Eq. (41), is a long formula: ${ }^{\S}$

$$
\begin{aligned}
& h=\left(0.00126476 T_{0}+\left(\left(-3.63534 \times 10^{-7}-1.52814 \times 10^{-10} T_{0}\right) T_{0}^{2}\right) /\left(6.98812 \times 10^{-10} h^{\prime} T_{0}{ }^{2}-\right.\right. \\
& 6.44784 \times 10^{-8} T_{0}^{3}+3.33204 \times 10^{-14} h^{\prime} T_{0}^{3}-4.14183 \times 10^{-12} T_{0}^{4}+3.97192 \times 10^{-19} h^{\prime} T_{0}{ }^{4}+(4 \\
& \left(8.6561 \times 10^{-7}+3.63865 \times 10^{-10} T_{0}\right)^{3} T_{0}^{6}+T_{0}^{4}\left(\left(-6.44784 \times 10^{-8}-4.14183 \times 10^{-12} T_{0}\right) T_{0}+h^{\prime}\right. \\
& \left.\left.\left.\left(6.98812 \times 10^{-10}+\left(3.33204 \times 10^{-14}+3.97192 \times 10^{-19} T_{0}\right) T_{0}\right)\right)^{2}\right)^{1 / 2}\right)^{1 / 3}+0.264567\left(6.98812 \times 10^{-10} h^{\prime}\right. \\
& T_{0}^{2}-6.44784 \times 10^{-8} T_{0}^{3}+3.33204 \times 10^{-14} h^{\prime} T_{0}^{3}-4.14183 \times 10^{-12} T_{0}^{4}+3.97192 \times 10^{-19} h^{\prime} T_{0}^{4}+(4 \\
& \left(8.6561 \times 10^{-7}+3.63865 \times 10^{-10} T_{0}\right)^{3} T_{0}^{6}+T_{0}^{4}\left(\left(-6.44784 \times 10^{-8}-4.14183 \times 10^{-12} T_{0}\right) T_{0}+h^{\prime}\right. \\
& \left.\left.\left.\left.\left(6.98812 \times 10^{-10}+\left(3.33204 \times 10^{-14}+3.97192 \times 10^{-19} T_{0}\right) T_{0}\right)\right)^{2}\right)^{1 / 2}\right)^{1 / 3}\right) /\left(5.08743 \times 10^{-6}+\right. \\
& \left.1.21288 \times 10^{-10} T_{0}\right)
\end{aligned}
$$

While leaving $T_{0}$ as an input parameter, Equation (42) cannot be simplified further using a truncated Taylor series in $h^{\prime}$ [as was done to obtain Eq. (38)]. It may be possible to eliminate some of the terms as negligible but this exercise is left to the reader. Despite its length, Eq. (42) remains a workable formula for coding and executing on computer. Alternatively, one could apply a series of sea-level temperatures to Eq. (41) and Eq. (42) in order to produce a series of functions in $h^{\prime}$ alone. Furthermore, the resulting series of inverse functions [i.e. those derived from Eq.(41)] could be further simplified using a truncated Taylor series. The resulting coefficients for the series of formulas could then be stored in a look-up table. This alternative would require significantly less computation at the cost of additional memory.

2. Adding Winds

The model presented also does not include winds. Winds speeds are true speeds that are often modeled as functions of altitude, i.e. W(h). Thus, in the presences of winds, Eq. (23) changes to:

$$
V_{T A S}=\left(\frac{K T}{f p s}\right) \frac{d r}{d t}+W(h)=\left(\csc \gamma \frac{K T}{f p s}\right) \frac{d h}{d t}+W(h)=V_{E A S} f(h)
$$

\footnotetext{
${ }^{\S}$ As solved and simplified by Mathematica ${ }^{\mathrm{TM}}$ Release 8.0
} 


$$
\int d t=t=\left(\csc \gamma \frac{K T}{f p S}\right) \int \frac{1}{\left(V_{E A S} f(h)-W(h)\right)} d h
$$

The integral for dh can be estimated using a Taylor series expansion as was done for Eq. (24). However, a similar algebraic formulation cannot be derived for the deceleration segment because the time and altitude terms cannot be segregated into separate integrals:

$$
\begin{gathered}
V_{T A S}=\left(\csc \gamma \frac{K T}{f p S}\right) \frac{d h}{d t}+W(h)=\left(V_{E A S}^{0}+a_{E A S} t\right) f(h) \\
\left(\left(V_{E A S}^{0}+a_{E A S} t\right) f(h)-W(h)\right) d t=\left(\csc \gamma \frac{K T}{f p S}\right) d h
\end{gathered}
$$

If one makes a simplifying assumption that the wind speed is an equivalent speed rather than a true speed, Eq. (44) and Eq. (46) change to:

$$
\begin{gathered}
t=\left(\csc \gamma \frac{K T}{f p S}\right) \int \frac{1}{\left(V_{E A S}-W(h)\right) f(h)} d h \\
\left(V_{E A S}^{0}+a_{E A S} t-W(h)\right) f(h) d t=\left(\csc \gamma \frac{K T}{f p S}\right) d h
\end{gathered}
$$

Again, the time and altitude terms for the deceleration segment cannot be segregated into separate integrals except in the case where the wind speed is constant with altitude. In this case, the resulting equations are identical to Eq. (24) to Eq. (34) with $\left(\mathrm{V}_{\mathrm{EAS}}-\mathrm{W}\right)$ substituted for $\mathrm{V}_{\mathrm{EAS}}$. Therefore, this is the only option for generating an algebraic formulation that includes winds.

3. Adapting the Model to Offset Runways

As previously discussed, formulation of the models as functions of altitude and glideslope angle facilitate the model's adaptation to runway pairs whose approach geometries may differ by threshold crossing height, runway elevation, final approach fix altitude, and glideslope angle. Accommodating runways that are offset in the longitudinal direction requires further adjustment because the equations for the procedure termination altitudes [Eq. (4) and Eq. (6)] implicitly assume no offset in the x-axis origin between runways. These equations derive from the assumption that the procedure ends when an aircraft crosses the threshold and that longitudinal location is the same whether the slow or fast aircraft crosses the threshold first. With offset runways, the procedure termination altitudes, $\mathrm{h}_{\mathrm{PTS}}$ and $\mathrm{h}_{\mathrm{PTF}}$, now depend on which aircraft is expected to cross the threshold first. The paired-approach without passing is the simpler case because the slow aircraft is always expected to cross the threshold first. For this case, $\mathrm{h}_{\text {PTS }}$ remains unchanged (using Eq. (4) where $\mathrm{D}_{\mathrm{ws}}$ is zero). However, Eq. (6) must be modified to include the runway offset distance $\left(\mathrm{D}_{\text {offset }}\right)$ in determining $\mathrm{h}_{\mathrm{PTF}}$ :

$$
h_{P T F}=h_{T C H}+\left(D_{C S}+D_{\text {offset }}\right) \tan \gamma
$$

However, in the case that allows passing, the fast aircraft can cross the threshold first only if the runway offset distance $\left(D_{\text {offset }}\right)$ is shorter than wake-safe distance $\left(D_{w s}\right)$. Otherwise, the slow aircraft must cross the threshold first if it is to avoid a wake encounter after the first aircraft passes. In this case, the fast aircraft, though ahead of the slow aircraft, is also behind its runway threshold when the procedure terminates. In the case where the fast aircraft reaches the threshold first, $h_{\text {PTF }}$ remains unchanged (using Eq. (6) with $D_{C S}$ equal to zero) and Eq. (4) must be modified to account for $D_{\text {offset: }}$

$$
h_{P T S}=h_{T C H}+\left(D_{W S}-D_{o f f s e t}\right) \tan \gamma
$$

In the case where the slow aircraft must cross the threshold first, then $\mathrm{h}_{\text {PTS }}$ remains unchanged (using Eq. (4) where $D_{W S}$ is zero) and Eq. (6) is adjusted for the runway offset as follows: 


$$
h_{P T F}=h_{T C H}+\left(D_{\text {offset }}-D_{W S}\right) \tan \gamma
$$

The equations that compute the runway $\mathrm{x}$-axis distance of the fast aircraft, required separation at the FAF $\left(\mathrm{S}_{\mathrm{FAF}}\right)$, and longitudinal compression $\left(\mathrm{d}_{\text {compress }}\right)$ also need adjustments for offset runways. Since the separation of the fast aircraft relative to the slow is the quantity of interest, the recommended approach is to adjust the equations to use the runway coordinates for the slow aircraft as the reference. For all scenarios, $\mathrm{D}_{\text {offset }}$ is added to Eq. (10) in the TAS model and Eq. (40) in the EAS model. In the case of the paired approach without passing, no other adjustments are necessary. However, the TAS model requires additional adjustments for the paired approach with passing that depend on which aircraft is expected to cross the threshold first. If the fast aircraft will cross the threshold first, then the TAS model must also add $\mathrm{D}_{\text {offset }}$ to Eq. (14). If the slow aircraft will land first, then the TAS model adds the wake-safe distance $\mathrm{D}_{\mathrm{ws}}$ instead of $\mathrm{D}_{\text {offset }}$ to Eq. (14).

\section{F. Model Limitations in Addressing Aircraft Performance Variability}

The equations in this paper model ideal performance; however, the actual performance of aircraft varies. First, the model does not account for path deviation (lateral or along-path flight technical error) which can modify the flown distance (and therefore flight time) from FAF to touchdown. The model also does not directly address the variation in the actual altitude where the slow aircraft initiates deceleration. Late deceleration will shorten the compression distance; early deceleration will lengthen it. Nevertheless, one can use the model to examine the bounds of late or early deceleration by assigning the altitude of the late or early deceleration to the variable $\mathrm{h}_{\mathrm{FAF}}$ for the slow aircraft. Likewise, actual deceleration not only can vary from the ideal, but it isn't a square impulse; it ramps up and ramps down. As demonstrated by the comparison with a high-fidelity simulation in section III.D, variability of deceleration appears to have small effect. Even so, one can reformulate the equations to accept a user-defined constant acceleration as an input in order to explore the variation bounds for deceleration. This would require additional equations to determine how much of the true (TAS model) or $\mathrm{D}_{\mathrm{EAS}}$ (EAS model) distance from the FAF to the procedure termination height (PTS or PTF) is covered by the deceleration segment and how much remains to be flown under the final approach speed. These equations are necessary to compute both $t_{\text {slow }}$ and $t_{\text {I-fast }}$. The equations for $\mathrm{X}_{\mathrm{D} \text {-fast }}$ (TAS model) and $\mathrm{D}_{\text {fast }}(\mathrm{EAS}$ model) are already dependent on an assigned acceleration (that of the slow aircraft) and would not need modification. For example, to compute $t_{\text {slow }}$ with a user defined acceleration, Eq. (2), Eq. (4), and Eq. (5) would be replaced with

$$
\begin{gathered}
t_{\text {decel }}=\frac{\Delta V}{a_{\text {slow }}}=\frac{\left(V_{f}-V_{c}\right)}{a_{\text {slow }}} \\
D_{\text {decel }}=t_{\text {decel }} V_{\text {avg }}=t_{\text {decel }} \frac{\left(V_{f}+V_{c}\right)}{2}=\frac{\left(V_{f}^{2}-V_{c}^{2}\right)}{2 a_{\text {slow }}} \\
t_{\text {final }}=\frac{\left(D_{F A F \rightarrow P T S}-D_{\text {decel }}\right)}{V_{f}}=\frac{\left(D\left(h_{F A F}, \gamma\right)-D\left(h_{P T S}, \gamma\right)-D_{\text {decel }}\right)}{V_{f}} \\
t_{\text {slow }}=t_{\text {decel }}+t_{\text {final }}
\end{gathered}
$$

where $\mathrm{D}(\mathrm{h}, \gamma)$ is the function for the TAS model [see Eq. (1)] or the EAS model [see Eq. (24)] that converts altitude and glideslope angle to glidepath distance from seal level.

Because much of the distance from FAF to threshold is traveled independently, deviations in actual aircraft speed from the scheduled speed can have a large impact on the decrease in longitudinal separation between the aircraft. ${ }^{3}$ The TAS and EAS models are both derived using constant speeds and the EAS model, in particular, cannot be modified to inject time or distance dependent variations in speed during a run. However, one can assume that, over the course of the approach, the effects of the random component of speed variation largely cancel out and that only the bias affects outcome. Thus, one can explore the bounds on speed deviation by applying constant biases to $\mathrm{V}_{\mathrm{f}}$ and $\mathrm{V}_{\mathrm{c}}$ for the slow and fast aircraft. The worst-case decrease in separation occurs when the negative limit on the velocity bias is applied to the slow aircraft and the positive limit on velocity bias is applied to the fast aircraft. ${ }^{4}$ Reference 3 provides an example of applying velocity biases to the TAS model to determine the required separation at the FAF that 
is robust to velocity bias. Similarly, Ref. 4 provides an example of applying velocity biases to the EAS model to determine required separation at the FAF that is robust to velocity bias.

\section{Conclusions}

A set of algebraic formulas were derived to estimate the travel time and separation loss of aircraft participating in a paired approach when the speed schedule of those aircraft is specified using equivalent airspeeds. This model was validated against a high-fidelity model of a civil transport flying the same path under autopilot and autothrottle control. Estimates of time to travel from FAF to threshold generally matched the high fidelity simulation within \pm 0.4 seconds. This paper also presented extensions of the EAS kinematic model to incorporate differences in sea-level atmospheric density from standard day (using the sea-level atmospheric temperature as a model input) and to incorporate constant winds though this extension requires treating the wind speed as an equivalent speed rather than a true speed. However, the EAS model could not be extended, algebraically, to wind speed as a function of altitude. The model also assumes an ideal path and does not address the path deviations caused by flight technical error. Nevertheless, the model inputs or its equations can be adjusted to explore the deviation bounds in the altitude of deceleration start by the slow aircraft, deviation bounds on the average deceleration of each aircraft, and bounds on bias in following the speed schedule.

When the EAS model is compared to similar algebraic formulas derived from the assumption that speed schedule is flown as true airspeed (TAS model), the differences in estimates of travel time and separation loss are modest. In the examples presented here, travel time differs up to 4 seconds, and separation loss differs by up to a 100 feet. Nevertheless, the EAS model results are more conservative than the TAS model. Moreover, paired approaches demand ever tighter performance margins as they are applied to runways separated by as little as 700 feet. Under such performance constraints, these modest differences have the potential to be significant determinants of required aircraft navigation performance and feasible aircraft pairings. The equations also remain simple enough to implement in real-time decision support tools as a higher fidelity alternative to the TAS model.

\section{References}

${ }^{1}$ Johnson, S. C., Abbott, T. S. , Guerreiro, N. M. G, Lohr, G. W., Volk, P., and McKissick, B. T., "Simplified Aircraft-Based Paired Approach: Concept Definition and Initial Analysis," National Aeronautics and Space Administration, NASA/TP-2013-217994, 2013.

${ }^{2}$ Bone, R., O. Olmos, and A. Mundra, "Paired Approach Operational Concept," Proceedings of the 20th Digital Avionics Systems Conference, Daytona Beach, FL, 20001.

${ }^{3}$ Perry, R. B., Madden, M. M., Torres-Pomales, W. and Butler, R. W., "Analysis and Simulation of the Simplified Aircraft-Based Paired Approach Concept With the ALAS Alerting Algorithm in Conjunction With Echelon and Offset Strategies," NASA/TM-2014-218151, 2014, publication pending.

${ }^{4}$ Torres-Pomales, W., Madden, M. M., Butler, R. W. and Perry, R. B., "The Simplified Aircraft-Based Paired Approach with the ALAS Alerting Algorithm,” NASA/TM-2013-217804, 2013.

${ }^{5}$ Hammer, J., "Case study of paired approach procedure to closely spaced parallel runways," Air Traffic Control Quarterly, Vol. 8(3), 223-252, 1999.

${ }^{6}$ Eftekari, R. R., Hammer, J. B., Havens, D. A., and Mundra, A.D., "Feasibility analyses for paired approach procedures for closely spaced parallel runways," Integrated Communications, Navigation and Surveillance Conference (ICNS), 2011 , pp. I5-1 - I5-14, 10-12 May 2011.

${ }^{7}$ Federal Aviation Administration, "Automatic Dependent Surveillance-Broadcast (ADS-B) Out Performance Requirements To Support Air Traffic Control (ATC) Service; Final Rule," Federal Register (National Archive and Records Administration) 75, no. 103, May 2010, pp. 30160 - 30195.

${ }^{8}$ National Oceanic and Atmospheric Administration, National Aeronautics And Space Administration, and United States Air Force, "U.S. Standard Atmosphere, 1976," NOAA-S/T 76-1562, U.S. Government Printing Office, Washington, D. C., 1976.

${ }^{9}$ Chung, V. I., and Hutchinson, B. K., "A Unique Software System For Simulation-To-Flight Research," AIAA Modeling and Simulation Technologies Conference \& Exhibit, Montreal, Canada, AIAA 2001-4057, August 6-9, 2001 